\title{
ON FINITE NILPOTENT GROUPS
}

\author{
G. BACHMAN
}

1. Introduction and notations. It is well known that if $(n, \phi(n))=1$, where $\phi(n)$ denotes the Euler $\phi$-function, then the only group of order $n$ is the cyclic group. This is a special case of a more general result due to Dickson (2, p. 201); namely, if

$$
n=p_{1}^{\alpha_{1}} \ldots p_{s}^{\alpha_{s}}
$$

where the $p_{i}$ are distinct primes and each $\alpha_{i}>0$, the necessary and sufficient conditions that the only groups of order $n$ are abelian are (1) each $\alpha_{i} \leqslant 2$ and (2) no

$$
p_{i}^{\alpha_{i}}-1
$$

is divisible by any $p_{1}, \ldots, p_{s}$.

We wish to establish a theorem which includes these two results. We let $G(n)$ equal the number of groups of order $n$ where

$$
n=\prod_{i=1}^{s} p_{i}^{\alpha_{i}}
$$

and we seek necessary and sufficient conditions on $n$ so that

$$
G(n)=\prod_{i=1}^{s} G\left(p_{i}^{\alpha_{i}}\right) .
$$

Clearly, this problem is equivalent to finding necessary and sufficient conditions on $n$ so that all existing groups of order $n$ be nilpotent.

It will be shown that the following is true:

THEOREM 1. Let

$$
n=p_{1}^{\alpha_{1}} p_{2}^{\alpha_{2}} \ldots p_{s}^{\alpha_{s}},
$$

where $p_{1}, \ldots, p_{s}$ are distinct primes and each $\alpha_{i}>0$. The necessary and sufficient conditions that the only groups of order $n$ be nilpotent are: $n_{0} p_{i}, i=1, \ldots, s$, shall divide any

$$
p_{j}^{\alpha_{j}}-1, p_{j}^{\alpha_{j-1}}-1, \ldots, p_{j}-1, j=1, \ldots, s .
$$

We introduce the following notations: the centre of a group $A$ by $Z(A)$, the group of all automorphisms of $A$ by $\Phi(A)$, the group of all inner automorphisms of $A$ by $\Phi^{\prime}(A)$, the factor group $\Phi(A) \mid \Phi^{\prime}(A)$ by $\mathfrak{2}(A)$, the direct

Received November 17, 1958. The author wishes to express his appreciation to Professor H. N. Shapiro for suggesting this problem to him. 
product of the two groups $A$ and $B$ by $A \times B$, and the direct product of the $n$ groups $A_{1}, \ldots, A_{n}$ by

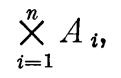

the order of the finite group $A$ by $|A|$, and a Sylow $p$-group of a group $G$ by $S_{p}$.

Let $B$ be a group such that each element $\alpha \in B$ is associated with an automorphism $a \rightarrow a^{\alpha}$ of $A$. Let $G$ be an extension of $A$ by $B$, that is, $A$ is a normal subgroup of $G$ and $G \mid A \simeq B$. Then the elements of $G$ can be written as $g_{\alpha} a$ where the $g_{\alpha}$ are in one-to-one correspondence with the $\alpha \in B$, and $a \in A$; also

$$
g_{\alpha} a \cdot g_{\beta} b=g_{\alpha \beta} f(\alpha, \beta) a^{\beta} b
$$

where $f(\alpha, \beta)$ is a factor system. Moreover,

$$
g_{\alpha}^{-1} a g_{\alpha}=a^{\alpha},
$$

and

$$
\left(a^{\alpha}\right)^{\beta}=\left(a^{\alpha \beta}\right)^{f(\alpha, \beta)} .
$$

Finally, to the extension $G$ there corresponds a well-defined homomorphism $\theta$ of $B$ into $\mathfrak{A}(A)$ (3, pp. 121-126). If $N$ is a normal subgroup of $G$ whose order is prime to its index, then $G$ splits over $N$ (Schur's theorem) (4, p. 132).

In general, if $A$ is abelian, then $\mathfrak{U}(A)=\Phi(A)$ and $B$ is a group of operators for $A$, that is, $A$ is a $B$-module. It is well known that the second cohomology group $H^{2}(B, A)$ is the group of all group extensions of $A$ by $B$. If $A$ and $B$ are finite and $(|B|,|A|)=1$, then $H^{r}(B, A)=0$ for all $r(\mathbf{1}$, p. 237), in particular, $H^{2}(B, A)=0$, so the only extensions of $A$ by $B$ are splitting extensions, that is, we can take $f(\alpha, \beta)=1$, and, therefore, $G$ contains a subgroup $B^{\prime} \simeq B$ such that $A \cap B^{\prime}=e$, the identity element, and $G=A B^{\prime}$.

The consideration of non-abelian groups $A$ is reduced to the abelian case by the following theorem: There exists a one-to-one correspondence between all non-equivalent extensions of $A$ by $B$ associated with $\theta$ and all non-equivalent extensions of $Z(A)$ by the group of operators $B$ corresponding to the homomorphism $\theta$ (3, pp. 142-145).

In the case of an abelian group $A$, the non-equivalent splitting extensions of $A$ by $B$ are in one-to-one correspondence with the distinct homomorphisms of $B$ into $\Phi(A)$ (3, p. 149). The kernel of the homomorphism will be denoted by $W$. If $W=B$, then we say that $B$ acts trivially on $A$.

2. Proof of Theorem 1. (1) Sufficiency: To proceed by induction, we assume that the statement is true for every

$$
n^{\prime}=p_{1}^{\beta_{1}} \ldots p_{s}^{\beta_{s}}, \beta_{i} \leqslant \alpha_{i}, n^{\prime}<n .
$$

Now since

$$
\left(|G|,\left(p_{i}^{\alpha_{i}}-1\right)\left(p_{i}^{\alpha_{i}-1}-1\right) \ldots\left(p_{i}-1\right)\right)=1
$$


we have, by Frobenius' Theorem (4, p. 143), that the maximal $p_{i}$-factor group of $G$ is isomorphic to every Sylow $p_{i}$-group of $G$, that is, $G$ contains a normal subgroup $N$ such that $G / N \simeq S_{p_{i}}$, and $G$ is a splitting extension of $N$. But there exists a one-to-one correspondence between all non-equivalent extensions of $N$ by $S_{p_{i}}$ associated with $\theta$ and all non-equivalent extensions of $Z(N)$ by the group of operators $S_{p_{i}}$ corresponding to the homomorphism $\theta$. Thus we must consider splitting extensions, $H$, of the $S_{p_{i}}$-module $Z(N)$. By the induction hypothesis,

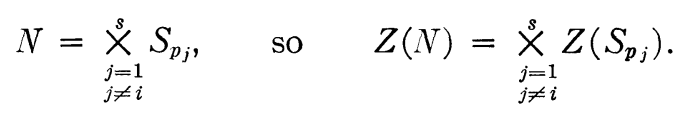

$Z\left(S_{p_{j}}\right)$ is an abelian group of order $p_{j}^{\gamma_{j}}, 1 \leqslant \gamma_{j} \leqslant \alpha_{j}$, from which it follows (4, p. 112) that $|\Phi(Z(N))|$ is a divisor of

$$
\prod_{\substack{j=1 \\ j \neq i}}^{s}\left(p_{j}^{\gamma_{j}}-1\right)\left(p_{j}^{\gamma_{j}}-p_{j}\right) \ldots\left(p_{j}^{\gamma_{j}}-p_{j}^{\gamma_{j}-1}\right) ;
$$

whence it is clear that we can only take $W=S_{p_{i}}$, which means trivial action, that is, the only extension of $Z(N)$ by $S_{p_{i}}$ is $S_{p_{i}} \times Z(N)$. Therefore, by the one-to-one correspondence there is only one extension of $N$ by $S_{p_{i}}$ associated with a given homomorphism $\theta$. Thus, the non-equivalent extensions of $N$ by $S_{p_{i}}$ are in one-to-one correspondence with those homomorphisms of $S_{p_{i}}$ into $\mathfrak{N}(\mathrm{V})$ which are associated with extensions of $N$ by $S_{p_{i}}$. But

$$
\Phi(N)=\underset{\substack{j=1 \\ j \neq i}}{\stackrel{s}{x}} \Phi\left(S_{p_{j}}\right) \quad \text { and } \quad|\Phi(N)|
$$

is a divisor of

$$
\prod_{\substack{j=1 \\ j \neq i}}^{s}\left(p_{j}^{\alpha_{j}}-1\right)\left(p_{j}^{\alpha_{j}}-p_{j}\right) \ldots\left(p_{j}^{\alpha_{j}}-p_{j}^{\alpha_{j}-1}\right) .
$$

Hence $|\mathfrak{H}(N)|$ is also a divisor of this number. Therefore, it is clear that the only possible homomorphism is the trivial one which implies that the only extension of $N$ by $S_{p_{i}}$ is $S_{p_{i}} \times N$. But

$$
N=\underset{\substack{j=1 \\ j \neq i}}{\underset{x}{x}} S_{p_{j}}
$$

hence

$$
G=\underset{j=1}{\stackrel{s}{x}} S_{p_{j}}
$$

and $G$ is nilpotent.

(2) Necessity: Suppose some

$$
p_{i} /\left(p_{j}^{\alpha_{j}}-1\right) \ldots\left(p_{j}-1\right) .
$$

Then we consider the following arrangement. Let

$$
A\left(\alpha_{j}, p_{j}\right)=C_{p_{j}} \times C_{p_{j}} \times \ldots \times C_{p_{j}} \quad\left(\alpha_{j} \text { times }\right)
$$


where $C_{p_{j}}$ is the cyclic group of order $p_{j}$. We denote by $G\left(\alpha_{i}, p_{i}\right)$ a group of order $p_{i}{ }^{\alpha_{i}}$. Now, clearly,

$$
\Phi\left(\underset{\substack{j=1 \\ j \neq i}}{\stackrel{s}{j}} A\left(\alpha_{j}, p_{j}\right)\right)=\underset{\substack{j=1 \\ j \neq i}}{\stackrel{s}{s}} \Phi\left(A\left(\alpha_{j}, p_{j}\right)\right)
$$

Since, by assumption,

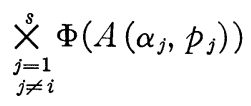

contains a subgroup of order $p_{i}$, there exists a homomorphism:

$$
G\left(\alpha_{i}, p_{i}\right) \rightarrow \Phi\left(\underset{\substack{j=1 \\ j \neq i}}{\stackrel{s}{j}} A\left(\alpha_{j}, p_{j}\right)\right)
$$

with kernel a normal subgroup $W$ of order $p_{i}^{\alpha_{i}-1}$. Associated with this homomorphism, there is a splitting extension $G$ of

$$
\underset{\substack{j=1 \\ j \neq i}}{\stackrel{s}{j}} A\left(\alpha_{j}, p_{j}\right) \quad \text { by } G\left(\alpha_{i}, p_{i}\right)
$$

for which $W$ is the normal subgroup of $G\left(\alpha_{i}, p_{i}\right)$ which acts trivially on

$$
\underset{\substack{j=1 \\ j \neq i}}{s} A\left(\alpha_{j}, p_{j}\right) .
$$

$G$ is, of course, a group of order

$$
n=\prod_{i=1}^{s} p_{i}^{\alpha_{i}}
$$

but the extension $G$ is not equivalent to

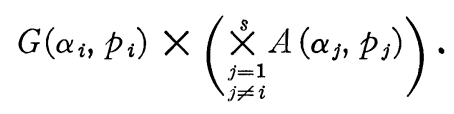

In fact, $G$ is not isomorphic to this group for $S_{p_{i}}=G\left(\alpha_{i}, p_{i}\right)$ is not normal in $G$. Namely, if $a^{-1} g_{\alpha} a=g_{\beta}$ then $g_{\alpha}^{-1} a^{-1} g_{\alpha} a=g_{\alpha}{ }^{-1} g_{\beta}$, that is, $\left(a^{-1}\right)^{\alpha} a=g_{\alpha}{ }^{-1} g_{\beta}$, but the left side belongs to

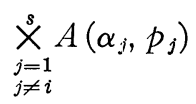

while the right side belongs to $S_{p_{i}}$. However,

$$
S_{p_{i}} \cap\left(\underset{\substack{j=1 \\ j \neq i}}{\stackrel{s}{j}} A\left(\alpha_{j}, p_{j}\right)\right)=e
$$

so $g_{\beta}=g_{\alpha}$. Now if $S_{p_{i}}$ were normal in $G$, then we would have $a^{-1} g_{\alpha} a=g_{\alpha}$ for all $g_{\alpha} \in S_{p_{i}}$ and all

$$
a \in \underset{\substack{j=1 \\ j \neq i}}{\stackrel{s}{y}} A\left(\alpha_{j}, p_{j}\right)
$$


that is $\left(a^{-1}\right)^{\alpha}=a^{-1}$ for all $\alpha$ and all $a$, which implies that $S_{p_{i}}$ acts trivially on

$$
\underset{\substack{j=1 \\ j \neq i}}{s} A\left(\alpha_{j}, p_{j}\right), \text { or } W=S_{p_{i}},
$$

which is a contradiction.

Therefore, $S_{p_{i}}$ is not normal in $G$; hence $G$, of order $n$, is not nilpotent.

Corollary 1. Let $G(n)$ be the number of groups of order $n$. If

$$
n=\prod_{i=1}^{s} p_{i}^{\alpha_{i}}
$$

then the necessary and sufficient conditions in order that

$$
G(n)=\prod_{i=1}^{s} G\left(p_{i}^{\alpha_{i}}\right)
$$

are that no $p_{i}, i=1,2, \ldots, s$, divides any

$$
\left(p_{j}^{\alpha_{j}}-1\right)\left(p_{j}^{\alpha_{j}-1}-1\right) \ldots\left(p_{j}-1\right) .
$$

Proof. There are $G\left(p_{i}^{\alpha_{i}}\right)$ groups of order $p_{i}^{\alpha_{i}}$. By taking all possible direct products it is clear that

$$
G(n) \geqslant \prod_{i=1}^{s} G\left(p_{i}^{\alpha_{i}}\right),
$$

and we have equality, if and only if the only groups of order $n$ are direct products of their Sylow subgroups.

It is clear that to have only abelian groups of order $n$, we must have $\alpha_{j} \leqslant 2$ for all $j$; hence we get Dickson's theorem as a special case of Theorem 1 .

\section{RefERENCES}

1. H. Cartan and S. Eilenberg, Homological algebra (Princeton, 1956).

2. L. E. Dickson, Trans. Amer. Math. Soc. 6 (1905), 201.

3. A. G. Kurosh, The theory of groups, vol. II (New York, 1956).

4. H. Zassenhaus, The theory of groups (New York, 1949).

Rutgers University 